\title{
PRIMARY SHOCK CALIBRATION WITH FAST LINEAR MOTOR DRIVE
}

\author{
H. Volkers ${ }^{1}$, H. C. Schoenekess ${ }^{1}$, Th. Bruns ${ }^{1}$ \\ ${ }^{1}$ Physikalisch-Technische Bundesanstalt, Braunschweig, Germany, henrik.volkers@ptb.de
}

\begin{abstract}
:
This paper describes the implementation of a new, fast and precise linear motor drive for PTB's primary shock calibration device. This device is used for monopole shock calibrations of accelerometers using the "hammer-anvil" principle according to ISO 16063-13:2001 [1] and operates in a peak acceleration range from $50 \mathrm{~m} / \mathrm{s}^{2}$ to $5000 \mathrm{~m} / \mathrm{s}^{2}$.

The main challenge of implementing this kind of shock generator is accelerating a hammer to velocities up to $5 \mathrm{~m} / \mathrm{s}$ within distances of less than $70 \mathrm{~mm}$.

In this paper, a few helpful improvements are described which lead to an enhanced repeatability of pulse generation over the full shock intensity range as well as a substantial decrease of harmonic disturbing signals.
\end{abstract}

Keywords: Primary shock calibration; hammeranvil principle; half-sine pulse; pulse transmission; LDV interferometry; linear motion drive; magnetic actuator

\section{INTRODUCTION}

PTB's original shock exciter which was designed for low to medium acceleration intensities mainly consists of a mechanical spring unit, an airborne transmission element (the "hammer") and an air-borne measuring unit (the "anvil") to which the accelerometer to be calibrated is attached. The released spring pushes the hammer which subsequently strikes the anvil and accelerates it. Exchangeable elastic pads (pulse shapers) between hammer and anvil allow certain shock shapes to be attained and disturbing oscillations to be reduced. Using the spring drive unit, shock peak values can be varied between $100 \mathrm{~m} / \mathrm{s}^{2}$ and $5000 \mathrm{~m} / \mathrm{s}^{2}$, whereas the shock duration is between $8 \mathrm{~ms}$ and $1 \mathrm{~ms}$. Different sets of the modules (hammer, anvil and spring unit) are available, allowing different combinations of peak values and shock durations to be excited.

The acceleration is measured by applying two laser Doppler vibrometers (LDV). After a signal conditioning, both photo detector signals and the sensor measuring chain signal are simultaneously captured. Following the signal demodulation, the primary measured acceleration peak and the sensor peak are used to determine the shock sensitivity according to

$S_{\text {sh }}=\frac{u_{\text {peak }}}{a_{\text {peak }}}$

By definition [1], the shock sensitivity $S_{\mathrm{sh}}$ is calculated as the quotient of the output charge peak value of the accelerometer and the peak value of the interferential measured shock acceleration (evaluation in the time domain). It is dependent on the impact spectrum, the duration of impact and the peak acceleration value.

\section{THE INITIAL CONFIGURATION}

The original driving mechanism to accelerate the hammer was realized by a spring-driven shaft with a free running length of up to $30 \mathrm{~mm}$ (c.f. Figure 1). By manually setting the spring preload, the resulting hammer velocity was adjusted to generate the desired peak acceleration of the anvil.

Repeated loading and releasing of the spring was performed by motor-driven mechanics. A set of three spring modules with different stiffnesses provides the peak acceleration ranges needed.

All of these spring units suffered from substantial drawbacks such as:

- The highest force acts at the start of motion, which results in a strong jerk. This introduces high frequency vibrations and excites resonances in the hammer and the anvil.

- The mechanical stop of the spring unit induces additional vibrations to the system.

- The force's set-point has to be mechanically adjusted by hand.

- The repeatability of adjusted shock intensities is of only moderate quality.

- Three different spring units have to be employed to cover the $100 \mathrm{~m} / \mathrm{s}^{2}$ to $5 \mathrm{~km} / \mathrm{s}^{2}$ range.

- Shock intensities below $100 \mathrm{~m} / \mathrm{s}^{2}$ could not be achieved with the old spring units. 
- Mechanical wear of the springs, locking knife edges and the release mechanism required frequent maintenance and readjustment. These were the main causes of poor repeatability.

- The calibration process involved a lot of manual interaction.

The design and realization of this unit was the first of this type and was done in the $1990^{\text {th }}$ of the last century $[2,3]$. Other NMIs have set up similar devices in the meantime, sometimes with different drive methods [e.g. 4,5]. To address the issues listed above, a project was started to update the driving unit specifically.

The complete mechanical set-up of the calibration device is shown in Figure 1. The manually adjustable spring unit is at the bottom. The air-borne hammer (the projectile) in the centre has a rectilinear motion distance of roughly $50 \mathrm{~mm}$ and is stopped after the impact with the anvil by mechanical dampers. The air-borne anvil (the target) at the top of the photograph has a freemotion distance of about $8 \mathrm{~mm}$ before it is halted by a pneumatic brake.

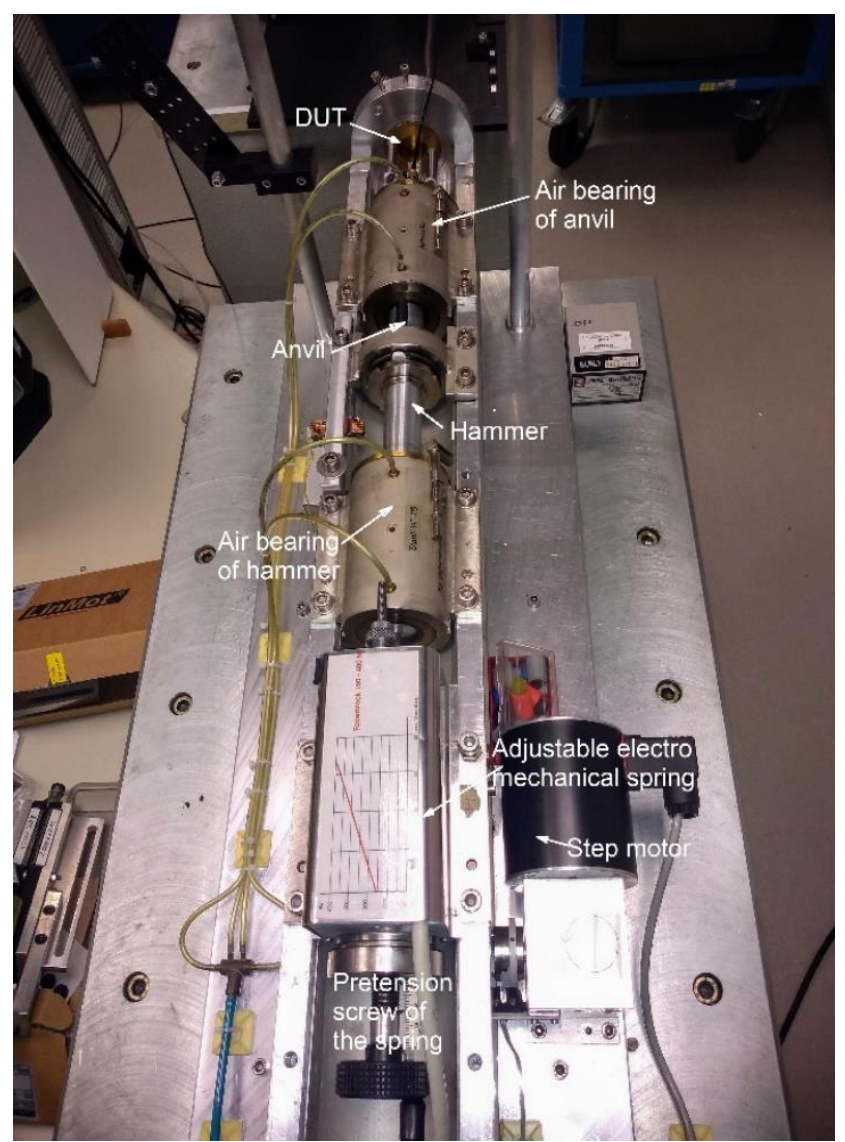

Figure 1: Mechanics of the shock calibration device

The impulse hammer element with a mass of approx. $0.25 \mathrm{~kg}$ hits the $0.25 \mathrm{~kg}$ anvil element with the mounted accelerometer $(0.038 \mathrm{~kg})$. The shock pulse is transmitted within a time that is shorter than 8 milliseconds. When the shortest duration (roughly $1 \mathrm{~ms}$ ) is used, high accelerations can be achieved.
The strongest spring with a maximum force of $400 \mathrm{~N}$ achieved more than $5 \mathrm{~km} / \mathrm{s}^{2}$ at the sensor's reference surface, while the softest spring, with a minimum preload force of about $10 \mathrm{~N}$, reached $100 \mathrm{~m} / \mathrm{s}^{2}$.

Via a suitably elastic pulse shaper at the tip of the hammer, a $\sin ^{2}$ acceleration time curve is generated and sensed by the accelerometer (c.f. Fig. 2).

Several different pulse shapers made of rubber could be attached to the hammer to obtain the desired pulse duration and shape.

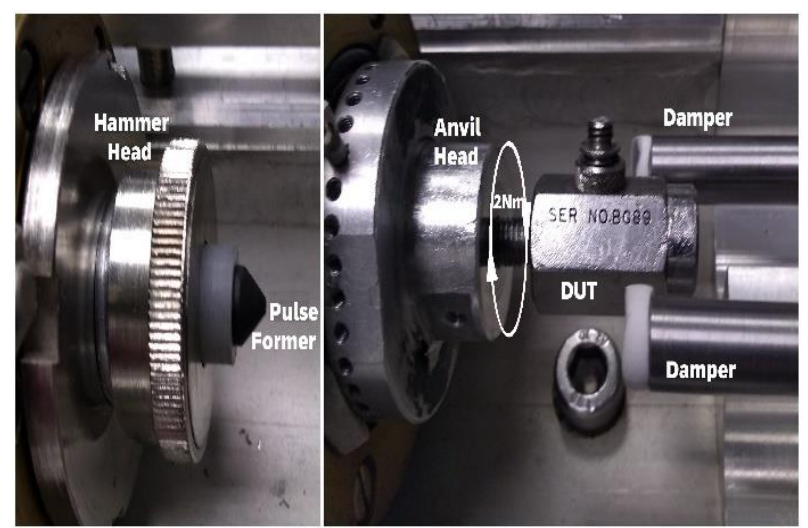

Figure 2: Hammer and anvil heads with DUT

As discussed above, the mechanical spring unit proved to be a weak point in the old design. During the relaxation of the spring, strong vibrations were transmitted into the entire mechanical system, which led to disturbances. In addition, the knifeedge mechanics of the release mechanism suffered from wear and tear and caused a poor repeatability during calibration runs.

The goal of this project was to overcome these issues using modern linear drive technology.

\section{THE NEW DRIVE UNIT}

\subsection{Requirements and design}

In order to ensure the full operation of the customer calibration service, it was necessary to have backstop conversion capability during the development and testing period. This resulted in dimensional design constraints.

By applying laws of basic mechanics, the required mechanical specifications were easily derived from the involved moving masses, impact speeds and dimensions.

Subsequent market research covering mechanic, pneumatic, hydraulic and electro-magnetic actuators led to the decision to use an electric linear motor drive.

The selected solution is a type PS01-23x160HHP-R linear motor with a PL01-12x350/310-HP magnetic slider as well as a type $\mathrm{C} 1100$ standard closed loop controller and a LinMot ${ }^{\circledR}$ NTI AG two- 
phase power supply ${ }^{1}[6]$. The package also includes software for the controller.

This kind of drive was originally designed for so-called "fast pick and place" operation in industrial production lines. The use of such industrial equipment means that only moderate hardware costs are involved.

In order to implement the drive, some modifications had to be applied to the calibration device such as:

- Construction of a damped stop for the runner of the linear motor (pusher).

- A prolongation of the hammer and an increase of the distance between hammer and anvil air bearings.

- An increase of the hammer's mass (c.f. Figure 3).

The last two actions permitted the working length of the drive to be enlarged by about $72 \mathrm{~mm}$. In the original set-up, similar hammer and anvil dimensions were chosen for an optimal momentum transfer during impact. This, however, also resulted in similar first modal resonances around $10 \mathrm{kHz}$, which in turn favoured the propagation of disturbing oscillations during impact. The new hammer design reduces this effect by means of a (two times) heavier weight.

Table 1 summarizes the modified parameters.

Table 1: Specifications of both impact generators

\begin{tabular}{|c|c|c|}
\hline Change & Old state & New state \\
\hline hammer weight & $0.296 \mathrm{~kg}$ & $0.595 \mathrm{~kg}$ \\
\hline anvil weight & $0.296 \mathrm{~kg}$ & $0.296 \mathrm{~kg}$ \\
\hline max. travel hammer & $50 \mathrm{~mm}$ & $72 \mathrm{~mm}$ \\
\hline max. travel anvil & 4 to $7 \mathrm{~mm}$ & 7 to $8 \mathrm{~mm}$ \\
\hline max. velocity hammer & $5 \mathrm{~m} / \mathrm{s}$ & $5 \mathrm{~m} / \mathrm{s}$ \\
\hline max. velocity anvil & $5 \mathrm{~m} / \mathrm{s}$ & 6 to $7 \mathrm{~m} / \mathrm{s}$ \\
\hline shock duration range & 1 to $8 \mathrm{~ms}$ & 1 to $8 \mathrm{~ms}$ \\
\hline max. drive force & $400 \mathrm{~N}$ & $138 \mathrm{~N}$ \\
\hline max. shock acceleration & $5000 \mathrm{~m} / \mathrm{s}^{2}$ & $5000^{+} \mathrm{m} / \mathrm{s}^{2}$ \\
\hline
\end{tabular}

These system modifications are a consequence of changing the hammer weight and travel parameters. The main improvements are found in the higher anvil velocity and, at the same time, the reduction of drive force to less than half. The latter greatly reduced the jerk and thus the issue of motion disturbances.

\subsection{Quasi-elastic central impact calculation}

The selected linear motor with a maximum propulsive force of $F_{M o t}=138 \mathrm{~N}$ serves as a new mechanical shock generator. The stroke $s$ has been extended to $72 \mathrm{~mm}$. The total mass to be accelerated consists of the runner's $0.280 \mathrm{~kg}$ mass $\mathrm{m}_{\mathrm{sl}}$ and the

\footnotetext{
${ }^{1}$ Commercial components are merely identified in this paper to adequately specify the experimental set-up. Naming these products does not imply a recommendation by PTB, nor does it
}

mass of the new air-borne hammer $\left(m_{\mathrm{Ha}}=\right.$ $0.595 \mathrm{~kg}$ ). The mass of the anvil to be pushed by the hammer is $m_{A n}=0.296 \mathrm{~kg}$.

The initial velocity reached by the accelerated hammer before impact is

$$
V_{H a}=\sqrt[2]{\frac{2 \cdot F_{M o t} S}{m_{s l}+m_{A n}}} .
$$

It attains up to $5 \mathrm{~m} / \mathrm{s}$.

The moving hammer $m_{H a}$ pushes the resting anvil $m_{A n}$ and transfers a large amount of its momentum to it.

The mass ratio is $m_{H a} / m_{A n}=2$ and the anvil is initially at rest.

Applying the basic equations for momentum conservation, the speeds of the hammer and anvil after the impact become:

$$
V_{H a}^{\prime}=\frac{m_{H a}-m_{A n}}{m_{H a}+m_{A n}} \cdot V_{H a}
$$

and

$$
V_{A n}^{\prime}=2 \cdot \frac{m_{H a}}{m_{H a}+m_{A n}} \cdot V_{H a}
$$

Or, with the given mass ratio:

$$
V_{H a}^{\prime}=\frac{1}{3} \cdot V_{H a} \text { and } V_{A n}^{\prime}=\frac{4}{3} \cdot V_{H a}
$$

This transformation moves the anvil to a onethird higher speed than the hammer's initial velocity.

For an optimal hammer mass, one could insert (2) into the right hand side of (4) and solve for the maximum value. This gives an optimum hammer mass of $0.581 \mathrm{~kg}$ when all boundary conditions are considered. This is pretty close to the realized new hammer.

Figure 3 in the next section shows the new shock generator setup.

\subsection{Repeatability}

An additional advantage of this linear motor drive set-up is the option of preselecting an accurate target value by setting a potentiometer or using the software interface for digital adjustment.

The controller of the linear motor can be parametrized and controlled via two serial interfaces by the supplied vendor software or a freely available LabVIEW driver which can be adapted to individual needs.

For any given setting, the spread of the repeatedly realized acceleration peak values was drastically reduced compared to the original spring drive module. A comparison is depicted in Figure 4. For both drives, the absolute range around the nominal value increases linearly with the shock

imply that the equipment identified is necessarily the best available for the purpose. 
intensity. However, for the new linear drive, the spread is more than an order of magnitude lower than before.

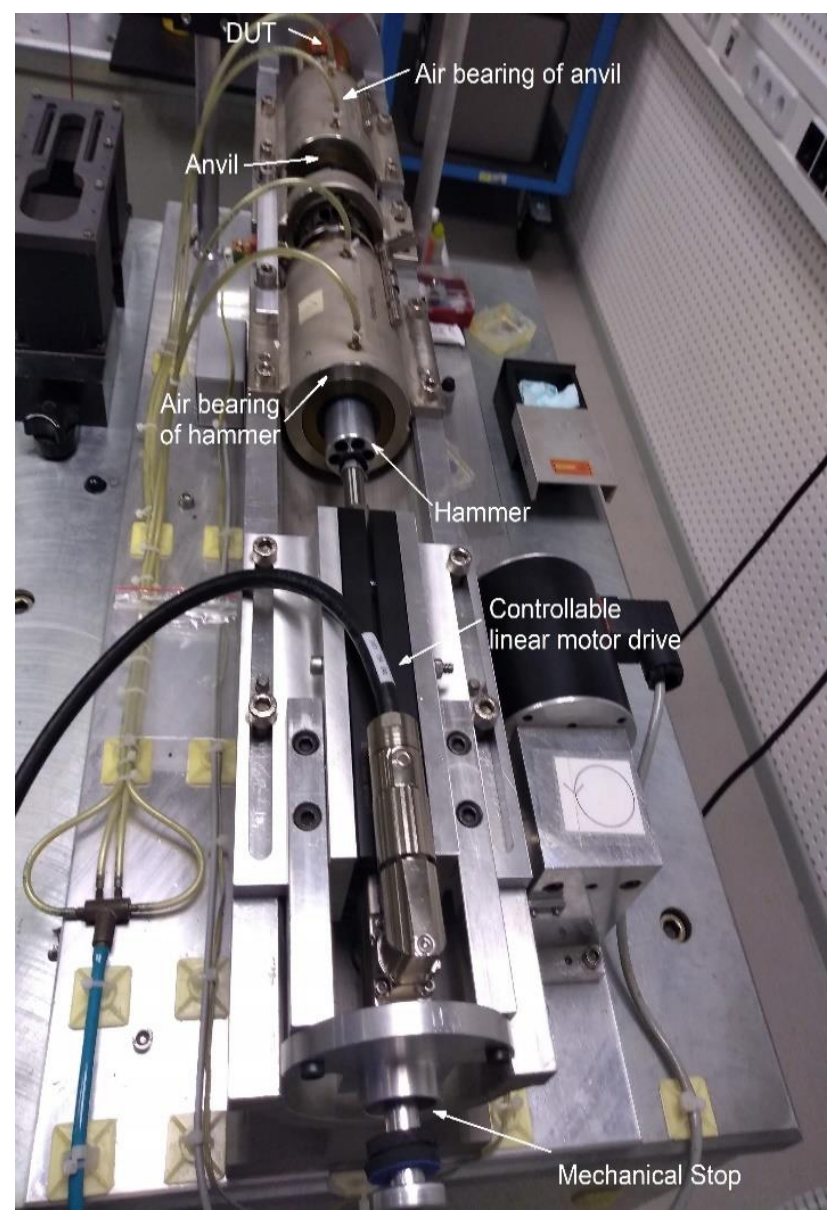

Figure 3: Shock generator for $5000 \mathrm{~m} / \mathrm{s}^{2}$ with fine controllable linear motor drive

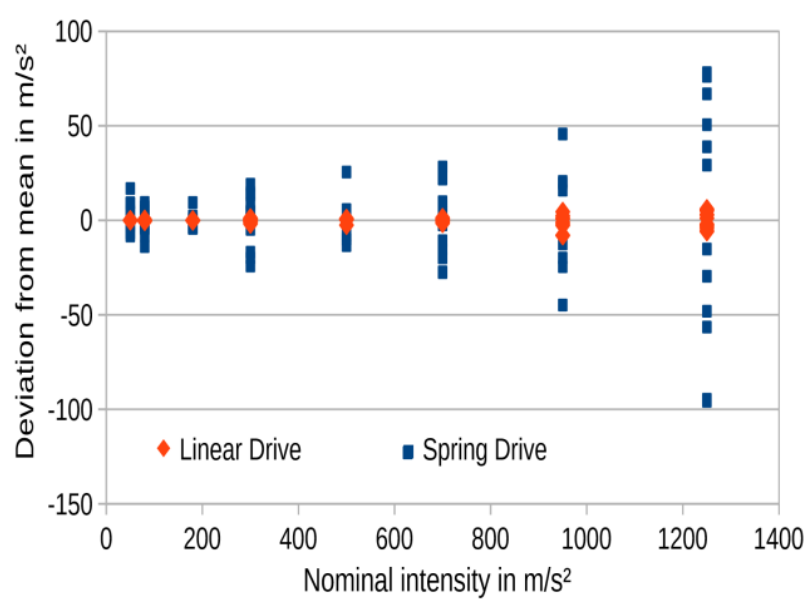

Figure 4: Comparison of the statistical reach of acceleration intensity of spring drive vs. linear motor drive

\subsection{Signal quality}

The disturbances due to modal resonances of the anvil are reduced by the redesign of the hammer.

While the resonance frequency of both the hammer and anvil was about $11.5 \mathrm{kHz}$ in the old setup, the first longitudinal resonance of the new hammer is now at $13 \mathrm{kHz}$. Because the design and materials were identical, this difference can be attributed to the elongated geometry and the solid design (no axial holes). This disparity greatly reduces the transmission of ringing vibrations from the hammer to the reference surface of the accelerometer. In combination with the largely reduced jerk in the driving motion itself, ringing is no longer an issue in the signal shape.

As an example, Figure 5 shows an accelerometer output signal (filtered at $100 \mathrm{kHz}$ ) for an acceleration peak value of $1.97 \mathrm{~km} / \mathrm{s}^{2}$.

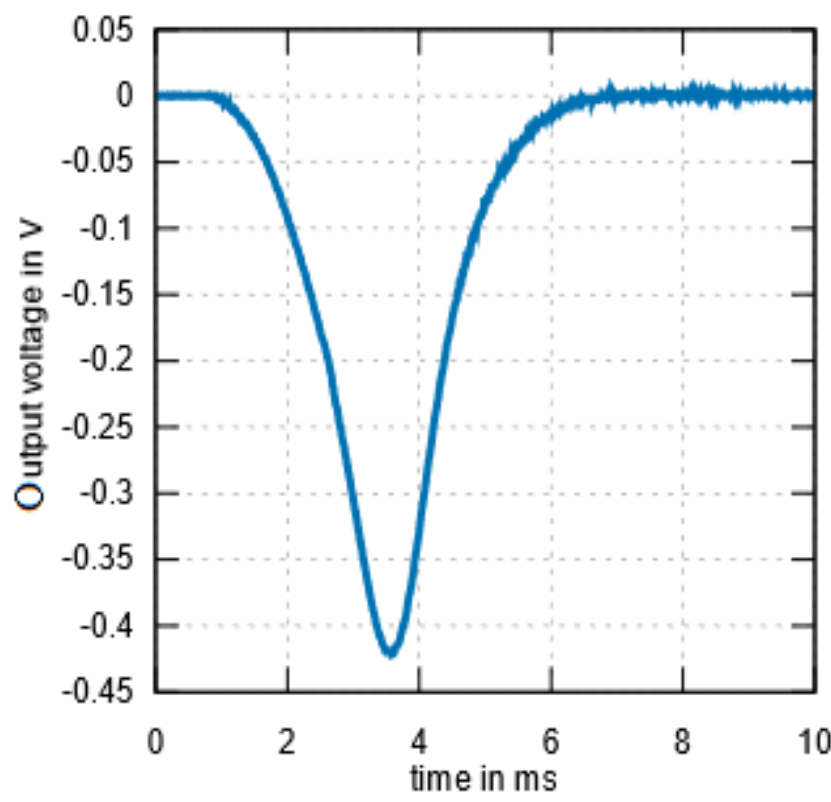

Figure 5: Raw signal of a half-sine shock measurement by an Endevco 2270 accelerometer

\section{SUMMARY}

The primary monopole shock acceleration calibration facility at PTB has been successfully upgraded by the implementation of an industrial electric linear motor which was implemented as the driving unit for the hammer. The change in the driving mechanics and geometry involved adaptations to the hammer and anvil configuration, too. After successful implementation the following improvements were noticeable:

1. A strong reduction in disturbing vibrations due to a lower, but constant, force level generated by the new drive.

2. A very strong reduction in the transfer of mechanical ringing from the hammer to the anvil due to the weight disparity between the two.

3. Great improvement of the repeatability of the shock intensity. 
4. Analog electrical or digital control of the set-point for the generated shock acceleration.

5. Greatly reduced need for maintenance and operation is practically non-wear and has very little friction.

\section{OUTLOOK}

Based on the newly implemented drive a shift from manual operation to an automated performance of the low-intensity shock calibration seems feasible in the near future. The control options via a digital interface (RS-485) using LabVIEW or other programming languages together with the excellent repeatability allow, in principle, unsupervised operation.

However, in the current set-up the pulse shaper remains as a component with unknown reproducibility. In order to achieve autonomous operation, some type of self-adjusting algorithm is necessary. First steps on the path towards such methods have been taken here and will be the topic of future publications.

\section{REFERENCES}

[1] ISO 16063-13, https://www.iso.org/standard/27075.html

[2] von Martens, H.-J. Taeubner A., Wabinski W., Link A., and. Schlaak H.-J "Laser interferometry as tool and object in vibration and shock calibrations", Proc. SPIE 3411, Third International Conference on Vibration Measurements by Laser Techniques: Advances and Applications, (1 June 1998); https://doi.org/10.1117/12.307700

[3] von Martens H.-J., "PTB vibration and shock calibration", Royal Swedish Academy of Engineering Sciences (IVA), 8 pages, 15-16 September 1993, Stenungsund, Sweden

[4] Nozato H., Kokuyama W., Ota A., "Improvement and validity of shock measurements using heterodyne laser interferometer", Elsevier Measurement, Volume 77, Pages 67-72, January 2016

[5] Nozato H., Usuda T., Ota A., Ishigami T. and Kudo K., "Development of Shock Acceleration Calibration Machine in NMIJ", IMEKO 20th TC3, 3rd TC16 and 1st TC22 International Conference Cultivating metrological knowledge 27-30 November, 2007. Merida, Mexico.

[6] LinMot Webpage https://linmot.com/ 